\title{
Deraumanas Analysis of queries from nurses to the South African National HIV \& TB Health Care Worker Hotline
}

\author{
A M Swart, ${ }^{1}$ BSc Pharm; B S Chisholm, ${ }^{1}$ BPharm; K Cohen, ${ }^{2}$ MB ChB, MMed (Clin Pharm), MSc (Epidemiology); \\ L J Workman, ${ }^{2} \mathrm{MPH}$ (Biostatistics \& Epidemiology); D Cameron, ${ }^{3,4} \mathrm{MB}$ ChB, MPraxMed, MPhil (Palliative Medicine), FCP (SA); \\ M Blockman, ${ }^{2} \mathrm{MB}$ ChB, BPharm, PG Dip Int Res Ethics, MMed (Clin Pharm) \\ ${ }^{1}$ Medicines Information Centre, University of Cape Town, Cape Town, South Africa \\ ${ }^{2}$ Division of Clinical Pharmacology, Department of Medicine, Faculty of Health Sciences, University of Cape Town, \\ Cape Town, South Africa \\ ${ }^{3}$ Foundation for Professional Development, Pretoria, South Africa \\ ${ }^{4}$ Department of Family Medicine, Faculty of Health Sciences, University of Pretoria, Pretoria, South Africa
}

Corresponding author: A M Swart (annoesjka.swart@uct.ac.za)

\begin{abstract}
Background. Since 2008, the Medicines Information Centre (MIC) has run the South African National HIV \& TB Health Care Worker Hotline which provides free information on patient treatment to all healthcare workers in South Africa. With the introduction of nurse-initiated management of antiretroviral therapy (NIMART) in the public sector, the need for easy access to HIV and tuberculosis (TB) information has increased, especially among nurses. The hotline aims to provide this, most importantly to nurses in rural areas, where clinical staff often have little access to peer review.

Objective. To describe the queries received from nurses by the hotline between 1 March and 31 May 2012 and identify problem areas and knowledge gaps where nurses may require further training.

Methods. All queries received from nurses during the study period were analysed. An experienced information pharmacist reviewed all queries to identify knowledge gaps.

Results. During the study period, the hotline received a total of $1479 \mathrm{HIV}$ - and TB-related queries from healthcare workers. Of these, 386 were received from nurses, of which 254 (66\%) were NIMART-trained. The most common query subtopic was initiating antiretroviral therapy (ART) (20\%), followed by adverse drug reactions (18\%). The most common knowledge gap identified was the ability to interpret laboratory results before initiating ART (10\%).

Discussion. We conclude that the hotline is providing clinical help to an increasing number of nurses on the topic of treating HIV and TB throughout South Africa. In addition, queries directed to the hotline may assist in identifying knowledge gaps for the further training of nurses.
\end{abstract}

S Afr J HIV Med 2013;14(4):179-182. DOI:10.7196/SAJHIVMED.948

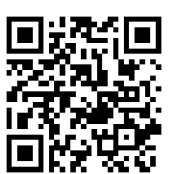

The Medicines Information Centre (MIC), situated within the Division of Clinical Pharmacology, Department of Medicine, Faculty of Health Sciences, University of Cape Town, is the only clinically based medicines information centre in South Africa (SA) and provides information to both public and private sector healthcare professionals. Established in 1980, the MIC's objective is to support rational prescribing in SA, by promoting the safe and effective use of medicines through the provision of unbiased, evidence-based information to its users.

SA healthcare workers see large numbers of HIV- and/or tuberculosis (TB)-infected patients. A large percentage of patients are HIV/TB co-infected, resulting in additional complexities. Many of these patients' care has been devolved to clinic level where they are managed by nurses. The management of such patients is often complicated, and the need for a clinical hotline to provide immediate advice is self-evident. Although the current hotline partially meets this need, expanded use is required to improve the care of HIV/TB-infected patients across SA.
Following the decision by the Minister of Health to expand the number of health facilities providing ART significantly in April $2010,{ }^{[1]}$ various institutions offered training to enable nurses to initiate and maintain patients on ART. These courses varied in length with standardised curriculum topics including HIV and $\mathrm{TB}$ diagnosis and management, interpreting laboratory results and clinical findings, ART regimens, clinical record-keeping and reporting. By the end of 2011, 10541 nurses had attended such a nurse-initiated management of antiretroviral therapy (NIMART) training course. ${ }^{[2]}$

After the theoretical component of the NIMART course, it was intended that these nurses would work under the supervision of an experienced mentor. In practice, however, regular mentor visits were not always possible, and many of the doctors who visited the clinics had themselves not received training in HIV/AIDS. ${ }^{[3]}$

In March 2008, in collaboration with the Foundation for Professional Development (FPD) and the US President's Emergency Fund for AIDS Reflief (PEPFAR)/US Agency 
for International Development (USAID), the MIC established the toll-free National HIV \& TB Health Care Worker Hotline, to provide information to all healthcare workers in SA on all aspects concerning the treatment of HIV and TB.

The hotline received 2035 calls between March 2008 and February 2009 (year 1) and 5449 between March 2011 and February 2012 (year 4) - a 2.6-fold increase. The percentage of calls from nurses increased from $8 \%$ during the first six months of the service in 2008 to $28 \%$ of total calls during the six months ending in February 2012 (Fig. 1).

Queries received by the hotline cover a range of topics including HIV testing, postexposure prophylaxis, the management of HIV in pregnancy, prevention of mother-tochild transmission (PMTCT) of HIV, when to initiate ART, regimen selection, laboratory and clinical monitoring, interpreting and responding to laboratory results, management of adverse events, drug interactions, treatment and prophylaxis of $\mathrm{TB}$ and opportunistic infections, drug availability and adherence support.

During many of the NIMART courses, nurses were encouraged to make use of the tollfree National HIV \& TB Health Care Worker hotline for advice with any drug-related problems.

The objectives of this study were: (i) to describe the queries received from nurses by the hotline between 1 March and 31 May 2012; and (ii) to identify knowledge gaps where nurses require further training.

\section{Methods}

The queries directed to the hotline are handled by four specially-trained drug information pharmacists. They have direct access to the latest information databases and reference sources and to a network of experienced clinicians and consultants across SA. Queries requiring clinical input are discussed with clinicians experienced in HIV management.

All queries received between 1 March and 31 May 2012 were included. Only queries received from nurses were analysed. Queries were recorded using standardised data-capture sheets and were entered into a Microsoft Access database.

The following was recorded:

- province

- whether or not the answer was given immediately

- when the answer was provided immediately, how long the telephone conversation lasted
- when not immediately answered, how long before the answer was given

- subtopic(s) of question in 25 pre-defined subject areas

- how many times the same nurses called the hotline during the study period

- how many queries received from nurses needed a specialist consultant's input

- whether or not the querying nurse had been NIMART-trained.

To identify specific gaps in the medical knowledge of nurses making use of the hotline, an experienced information pharmacist manually reviewed all the questions during the study period.

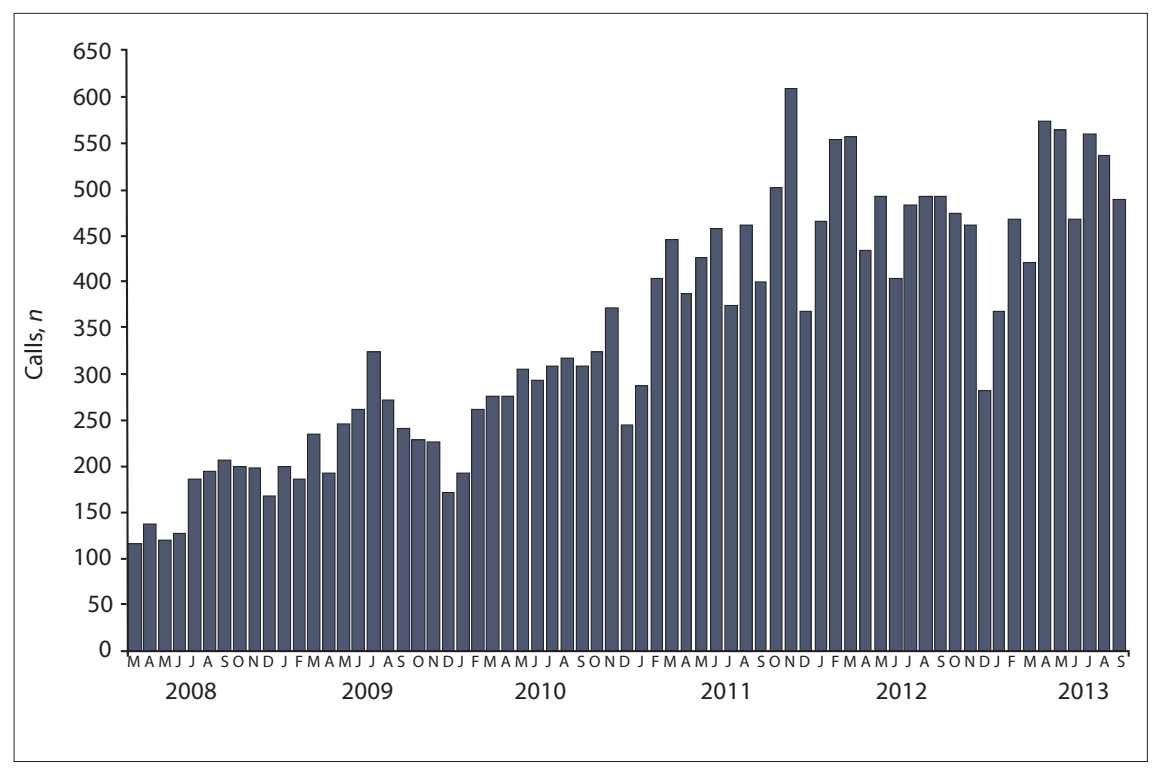

Fig. 1. Total number of calls per month to the hotline (all healthcare workers).

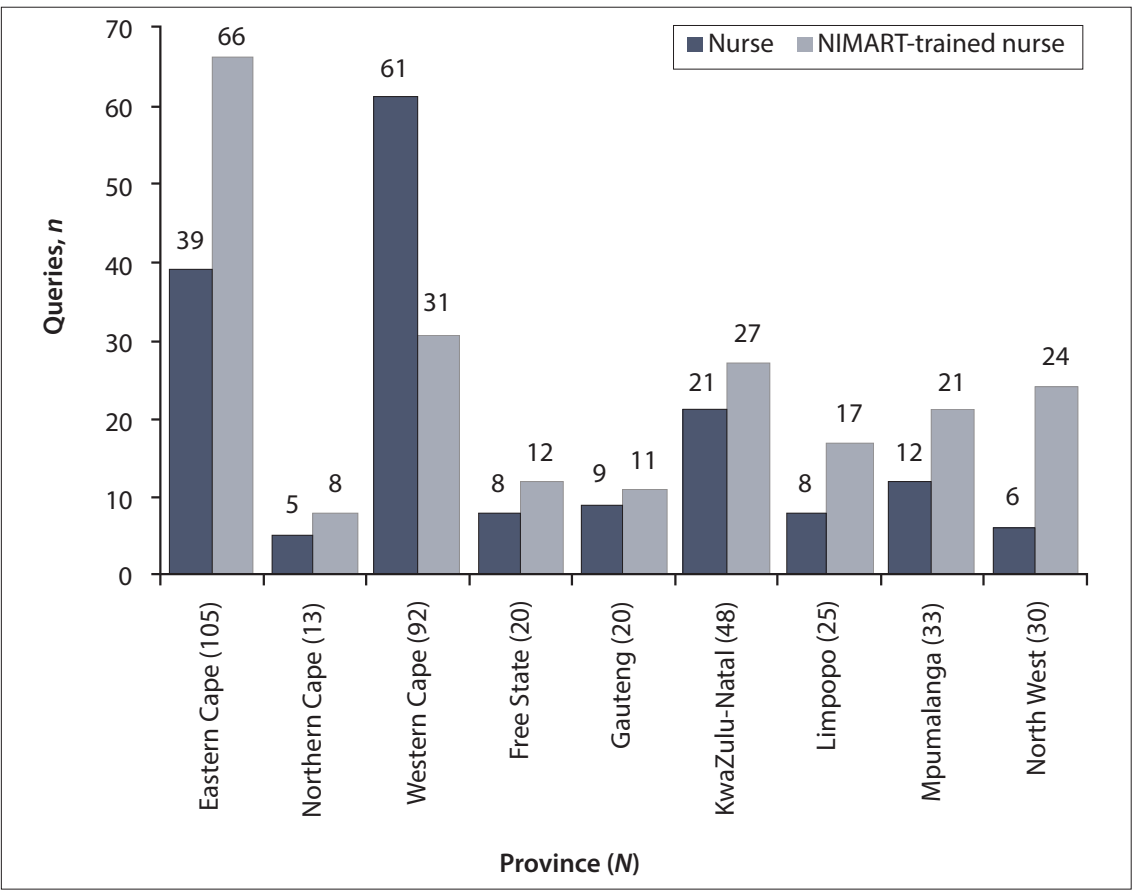

Fig. 2. Nurse calls by province. (NIMART = nurse-initiated management of antiretroviral therapy).

\section{Ethical considerations}

The study was approved by the Human Research Ethics Committee of the Faculty of Health Sciences, University of Cape Town. The confidentiality of patients was maintained throughout.

\section{Statistical analysis}

Associations between categorical variables were explored by cross-tabulation, using the $\chi^{2}$ test. Statistical significance was assumed at $p \leq 0.05$.

\section{Results}

During the study period, a total of 1479 HIVand TB-related queries were received by the

.

.


hotline from all healthcare professionals. Of these, 386 were received from nurses (26\%), including 254 (66\%) from nurses with NIMART training. The proportion of nurses who reported NIMART training differed significantly between provinces, with $<60 \%$ reporting NIMART training in Gauteng (55\%), Western Cape (34\%) and KwaZuluNatal (56\%).

Fig. 2 illustrates the breakdown of the calls received per province by NIMART-trained and non-NIMART-trained nurses. During the three-month study period, most of the calls from nurses were received from the Eastern Cape (27.2\%), followed by the Western Cape (23.8\%). Only 13 calls were received from the Northern Cape.

Of the 386 queries received, 278 (72\%) were answered immediately. Eighty-two queries (21\%) were answered within $30 \mathrm{~min}, 10$ queries (3\%) were answered between $30 \mathrm{~min}$ and 1 hour after receipt and 12 queries (3\%) took $1-4$ hours to answer. Only three queries (1\%) were not answered on the day that they were received.

The median time a call lasted when answered immediately was $5 \mathrm{~min}$ (range 0 - 18). There was no significant difference in the median time a call lasted between NIMART-trained nurses (median 4; interquartile range (IQR) 3 - 7) and non-NIMART-trained nurses (median 5; IQR 3 - 8 , rank-sum $p=0.69$ ).

\section{Subtopic(s) of questions}

The 386 queries were subcategorised into one or more of 25 subject areas (Table 1), with a total of 529 allocated subtopics. The two most common subtopics were initiating therapy (20\%) and adverse drug reactions (ADRs) (18\%). There was no significant difference between frequency of topic occurrence when comparing NIMARTtrained with non-NIMART-trained nurses $\left(\chi^{2}\right.$ $p=0.79$ ).

The frequency of query subtopics differed significantly between regions $\left(\chi^{2} p=0.003\right)$. Initiating therapy accounted for the highest proportion of query subtopics in KwaZuluNatal (37\%) and the lowest in the Western Cape (6\%). ADRs accounted for the highest proportion of query subtopics in the Western Cape $(23 \%)$ and the lowest in the Eastern Cape (5\%).

\section{Knowledge gaps for further training}

An experienced information pharmacist manually reviewed all the questions during the study period to identify specific gaps in the medical knowledge of nurses making use of the hotline.

For 182/386 queries (47\%), no specific knowledge gap was identified. For the remaining 204 queries (53\%), 22 different knowledge gaps were identified (Table 2). The most common question (37 queries) was how to interpret laboratory results before initiating patients on ART.

\section{Number of calls and per nurse}

During the study period, 214 nurses called 386 times (range 1 - 19 calls per nurse). Sixty-five (30\%) nurses called more than once, and 23 (11\%) called more than three times.

\section{Queries requiring a specialist consultant}

Of the 386 queries received, 59 (15\%) required the input of a medical doctor experienced in HIV medicine and could not be answered directly by the medicine information pharmacist who took the initial call.

\section{Discussion}

With about 1.8 million people receiving antiretroviral therapy (ART), SA has the world's largest public sector ART programme. ${ }^{[4]}$ Initially nurses were responsible for counselling, testing and preparing HIV-positive patients for ART. Since 2010, primary care nurses have also taken on the responsibility of initiating and maintaining patients receiving ART. This has resulted in increased access and reduced time to starting ART, reduced patient waiting times, improved patient satisfaction and comparable long-term outcomes. ${ }^{[5-7]}$ This task-shifting is supported by the SA National Department of Health and the World Health Organization, as well as the majority of health professionals both locally and internationally.

In general, NIMART-trained nurses phoned the hotline more than non-NIMART-trained nurses. This is probably due to the fact that nurses are made aware of the hotline during NIMART training.

The four most common subtopics were initiating therapy (20\%), ADRs (18\%), TB (7\%) and switching therapy (7\%). Further training of nurses in these areas may be necessary.

Seventy-two per cent of queries were answered immediately. Except for three queries which were not answered on the day that they were received, the remainder were answered within four hours of receipt. This indicates that, in most cases, the nurse can attend to the patient's needs immediately, which is critical in resource-poor, busy clinic environments.
Fifteen per cent of the queries received from nurses required the input of an infectious disease specialist and could not be answered directly by the medicine information pharmacist who took the initial call.

For 204/386 queries, an experienced drug information pharmacist identified important knowledge gaps that could guide the future training of nurses. The most common knowledge gap identified was a lack of understanding of how to interpret laboratory results before initiating patients on ART. Thirty-seven queries were identified with this specific problem. This has important ramifications for common ART prescribing areas and patient safety, e.g. administering tenofovir to patients with an estimated glomerular filtration rate (eGFR) $<50 \mathrm{ml} / \mathrm{min}$ and patients with liver function derangement.

The method of determining whether or not the nurse was NIMART-trained was limited by self report, i.e. it relied on the pharmacists

Table 1. Subtopic(s) of question

\begin{tabular}{|c|c|}
\hline Subtopic & $n(\%)^{*}$ \\
\hline Initiating therapy & $108(20)$ \\
\hline $\mathrm{ADR}$ & $94(18)$ \\
\hline $\mathrm{TB}$ & $37(7)$ \\
\hline Switching therapy & $36(7)$ \\
\hline Other & $34(6)$ \\
\hline Dosage & $33(6)$ \\
\hline Paediatrics & $32(6)$ \\
\hline Pregnancy & $27(5)$ \\
\hline Interactions & $24(5)$ \\
\hline Failure & $20(4)$ \\
\hline Adherence & $15(3)$ \\
\hline Second-line regimen & $13(2)$ \\
\hline OIs other than TB & $12(2)$ \\
\hline PEP & $12(2)$ \\
\hline PMTCT & $11(2)$ \\
\hline Availability/supply & $5(1)$ \\
\hline Lactation & $5(1)$ \\
\hline $\begin{array}{l}\text { Renal failure while receiving } \\
\text { TDF }\end{array}$ & $4(1)$ \\
\hline IRIS & $3(1)$ \\
\hline Resistance & $2(<1)$ \\
\hline Complementary medicines & $1(<1)$ \\
\hline Poisoning/overdose & $1(<1)$ \\
\hline \multicolumn{2}{|c|}{$\begin{array}{l}\mathrm{ADR}=\text { adverse drug reaction; } \mathrm{TB}=\text { tuberculosis; } \\
\text { OIs = opportunistic infections; } \mathrm{PEP}=\text { post-exposure } \\
\text { prophylaxis; } \mathrm{PMTCT}=\text { prevention of mother-to-child } \\
\text { transmission of HIV; TDF = tenofovir; IRIS = immune } \\
\text { reconstitution inflammatory syndrome. }\end{array}$} \\
\hline * Percentage of total subtopics allocated & 529). \\
\hline
\end{tabular}




\begin{tabular}{|c|c|c|c|}
\hline \multirow[b]{2}{*}{ Knowledge gap identified } & \multicolumn{3}{|c|}{$n(\%)$} \\
\hline & $\begin{array}{l}\text { NIMART- } \\
\text { trained nurse }\end{array}$ & Nurse & Total \\
\hline Unable to interpret laboratory results before initiating ART & $24(12)$ & $13(6)$ & $37(18)$ \\
\hline What ARVs to start in patients who have defaulted & $7(3)$ & $6(3)$ & $13(6)$ \\
\hline Use of EFV during pregnancy & $7(3)$ & $6(3)$ & $13(6)$ \\
\hline Initiating ART in children & $7(3)$ & $5(2)$ & $12(6)$ \\
\hline Initiating ART in patients with renal dysfunction (eGFR $<50 \mathrm{ml} / \mathrm{min}$ ) & $8(4)$ & $4(2)$ & $12(6)$ \\
\hline What ARV to change to when patients develop renal dysfunction while receiving TDF & $6(3)$ & $5(2)$ & $11(5)$ \\
\hline Paediatric dosing & $4(2)$ & $7(3)$ & $11(5)$ \\
\hline What to do when patients fail second-line ART & $7(3)$ & $3(1)$ & $10(5)$ \\
\hline How to deal with NVP rash or hepatitis & $6(3)$ & $3(1)$ & $9(4)$ \\
\hline Unable to calculate eGFR when serum creatinine value is available & $7(3)$ & $2(1)$ & $9(4)$ \\
\hline How to deal with patients who develop ADRs while receiving $\mathrm{d} 4 \mathrm{~T}$ & $5(2)$ & $3(1)$ & $8(4)$ \\
\hline $\begin{array}{l}\text { Duration of NVP treatment in babies (both breast- and formula-fed) on a PMTCT } \\
\text { programme (including those who have defaulted) }\end{array}$ & $2(1)$ & $6(3)$ & $8(4)$ \\
\hline Dosage adjustment of ARVs in patients with an eGFR $<50 \mathrm{ml} / \mathrm{min}$ & $6(3)$ & $2(1)$ & $8(4)$ \\
\hline Keeping patients on failing first-line ART for prolonged periods & $5(2)$ & $2(1)$ & $7(3)$ \\
\hline How to deal with drug stock-outs & $5(2)$ & $2(1)$ & $7(3)$ \\
\hline What to start in patients who are HBsAg-positive and/or have abnormal LFTs & $1(<1)$ & $5(2)$ & $6(3)$ \\
\hline Changing to non-interacting anticonvulsants before initiating or while receiving ART & $5(2)$ & $1(<1)$ & $6(3)$ \\
\hline Understanding the difference between serum creatinine and eGFR & $5(2)$ & $0(0)$ & $5(2)$ \\
\hline Indications for INH prophylaxis & $3(1)$ & $2(1)$ & $5(2)$ \\
\hline Preferred NNRTI in patients receiving TB treatment & $3(1)$ & $1(<1)$ & $4(2)$ \\
\hline Understanding why $\mathrm{CD} 4^{+}$counts do not increase in some patients who are receiving ART & $1(<1)$ & $1(<1)$ & $2(1)$ \\
\hline Age at which children can use TDF & $1(<1)$ & $0(0)$ & $1(<1)$ \\
\hline
\end{tabular}

asking the nurses during each call whether or not they were NIMARTtrained. In cases where this was not done, the nurse was classified as not NIMART-trained. We did not collect information on other training that nurses had received outside of the NIMART initiative.

\section{Conclusion}

The number of nurses contacting the hotline has increased significantly since its inception. This shows that the hotline is increasingly providing support to nurses throughout SA who are initiating and managing HIV and TB patients.

Author contributions. A Swart designed the study and managed the overall project. B Chisholm was responsible for the manuscript. D Cameron provided intellectual input and reviewed the manuscript. L Workman designed the database and performed statistical analysis. $\mathrm{K}$ Cohen performed statistical analysis and reviewed the manuscript. M Blockman reviewed the manuscript.

Acknowledgement. Sadly, Joe Talmud, who worked at the MIC since 1983, passed away in April 2013. He made many significant contributions to the safe and rational use of medicines during his career, while remaining humble and service-oriented. Improving the quality of life of the individual patient remained his ultimate goal. He stood for integrity, honesty and compassion. We will miss him dearly as a friend and colleague and acknowledge the contribution that he made to this article and to the hotline.

\section{References}

1. Motsoaledi A. Budget speech of the honourable Dr A Motsoaledi, Minister of Health, delivered to the National Assembly, Parliament of the Republic of South Africa, 13 April 2010. http://www.info.gov.za/speeches/2010/10041315551001.htm (accessed 6 November 2012).

2. Thabile Msila. Personal communication. National Department of Health (October 2012).

3. Georgeu D, Colvin CJ, Lewin S, et al. Implementing nurse-initiated and managed antiretroviral treatment (NIMART) in South Africa: A qualitative process evaluation of the STRETCH trial. Implement Sci 2012;7:66.

4. Mayosi BM, Lawn JE, van Niekerk A, et al. Health in South Africa: Changes and challenges since 2009. Lancet 2012;380:2029-2043. [http://dx.doi.org/10.1016/S01406736(12)61814-5]

5. Long L, Brennan A, Fox MP, et al. Treatment outcomes and cost-effectiveness of shifting management of stable ART patients to nurses in South Africa: An observational cohort. PLoS Med 2011;8(7):e1001055. [http://dx.doi.org/10.1371/journal.pmed.1001055]

6. Callaghan M, Ford N, Schneider H. A systematic review of task-shifting for HIV treatment and care in Africa. Hum Resour Health 2010;8:8. [http://dx.doi. org/10.1186/1478-4491-8-8]

7. Sanne I, Orrell C, Fox MP, et al. Nurse versus doctor management of HIVinfected patients receiving antiretroviral therapy (CIPRA-SA): A randomised non-inferiority trial. Lancet 2010;376:33-40. [http://dx.doi.org/10.1016/S01406736(10)60894-X] 